\title{
Home-Based Physical Activity in Patients With Type 2 Diabetes Mellitus: A Scoping Review
} Amirah Mustapa, MSc ${ }^{1}$, Maria Justine, $\mathrm{PhD}^{2,3}$, Aliff Abdul Latir, MSc ${ }^{2}$, Haidzir Manaf, $\mathrm{PhD}^{2,3}$

\author{
${ }^{1}$ Department of Physical Rehabilitation Sciences, Kulliyyah Allied Health Sciences, \\ International Islamic University Malaysia, Pahang; \\ ${ }^{2}$ Centre for Physiotherapy Studies, Faculty of Health Sciences, Universiti Teknologi MARA, Puncak Alam Campus, Selangor; \\ ${ }^{3}$ Clinical and Rehabilitation Research Group, Faculty of Health Sciences, Universiti Teknologi MARA, \\ Puncak Alam Campus, Selangor Darul Ehsan, Malaysia
}

To promote optimal healthcare delivery in type 2 diabetes mellitus (T2DM) following the outbreak of coronavirus disease 2019, adopting home-based physical activity (PA) is being seriously considered. Therefore, this study aims to outline the characteristics of exercise protocols for home-based PA and the challenges and limitations in implementing home-based PA in patients with T2DM. This scoping review was carried out by identifying eligible studies in six different databases (Scopus, Cochrane Library, SpringerLink, ProQuest, Science Direct, and Google Scholar). The keywords used in the search strategies were: home-based physical activity, homebased exercise therapy, home-based physical exercise, home-based exercise, home-based exercise training, diabetes mellitus, and T2DM. Two reviewers independently screened all full-text articles to find articles that met the eligibility requirements. A total of 443 articles were identified in the search. Approximately 342 articles were excluded by screening titles and abstracts, which led to the selection of 44 articles relevant to the current study. Further screening of the full-text led to the subsequent removal of 34 other articles, leading to 10 studies that were eligible for data extraction. This review suggested that the exercise protocols for home-based PA include resistance exercise using free weight and own body weight with a frequency of two to three sessions per week at moderate intensity, along with aerobic exercise (particularly walking) with a frequency of three to five times per week at moderate intensity. A combination of resistance and aerobic exercise showed more significant benefits of PA in patients with T2DM. More studies regarding home-based PA in T2DM patients with metabolic disorders are warranted.

Keywords Exercise, Physical activity, Resistance training, Diabetes mellitus type 2, Walking

Centre for Physiotherapy Studies, Faculty of Health Sciences, Universiti Teknologi MARA, Puncak Alam Campus, 42300, Puncak Alam, Selangor, Malaysia. Tel: +60126615246, E-mail: haidzir5894@uitm.edu.my

ORCID: Amirah Mustapa (https://orcid.org/0000-0002-7846-7669); Maria Justine (https://orcid.org/0000-0002-2412-8653); Aliff Abdul Latir (https:// orcid.org/0000-0001-8384-4070); Haidzir Manaf (https://orcid.org/0000-0003-0342-8136).

(c) This is an open-access article distributed under the terms of the Creative Commons Attribution Non-Commercial License (http://creativecommons.org/ licenses/by-nc/4.0) which permits unrestricted noncommercial use, distribution, and reproduction in any medium, provided the original work is properly cited. Copyright $\odot 2021$ by Korean Academy of Rehabilitation Medicine 


\section{INTRODUCTION}

Type 2 diabetes mellitus (T2DM) is a chronic and debilitating metabolic disease that accounts for $90 \%$ to $95 \%$ of all diabetes cases [1-3]. T2DM has a complex pathology, with hyperglycemia having a significant impact on metabolic and vascular factors, as well as genetic and environmental factors [4]. Driven by the impacts of prolonged hyperglycemia, numerous deleterious long-term complications on morbidity have been reported in diabetic patients [3-6]. Studies have shown that prolonged inactivity may significantly impact the pathogenesis of inflammation, which might explain the existence of substantial metabolic and cardiovascular complications in T2DM $[7,8]$.

In addition, the coronavirus disease 2019 (COVID-19) pandemic has emphasized the significant susceptibility in individuals with pre-existing comorbidities such as T2DM [9]. Driven by complex factors such as immune system deficiencies and hyperglycemia, studies have shown that individuals with pre-existing comorbidities are predisposed to the deleterious impacts of COVID-19, such as viral proliferation and severe inflammatory reactions of the upper respiratory tract $[9,10]$. Therefore, physical activity (PA) is constituted as a fundamental approach to combat the progressive pathologies of $\mathrm{CO}$ VID-19 in patients with T2DM $[9,10]$, prevent the complications of DM, and offer protection against the detrimental impacts of inactivity.

PA is defined as any unstructured bodily movement (self-selected leisure activity) that leads to greater energy expenditure than in rest $[11,12]$. The word of PA and exercise is used interchangeably. However, exercise is expressed as a structured PA (planned intentionally) which is intended to improve health and/or physical fitness $[11,12]$. Current international guidelines have recommended that diabetic patients should perform regular PA with at least 150 minutes of moderate-intensity or 75 minutes of vigorous-intensity aerobic exercise along with resistance exercise for three days per week $[3,13]$. Compared with the aerobic or resistance exercise alone, the combination of aerobic and resistance exercise is proven to have a synergistic and incremental effect on glycemic control as well as decrease risk of cardiovascular complications and overall mortality among T2DM patients $[9,12]$.
Studies have shown that regular PA among patients with T2DM in nationwide representative samples remains low to moderate, ranging from $30 \%$ to $60 \%$ [14-16]. Furthermore, current stringent lockdown orders following the COVID-19 pandemic have caused the majority of diabetic patients to become physically inactive [17-19]. In Poland, about $34 \%$ of 124 diabetic patients are inactive compared with $21 \%$ before the COVID-19 pandemic [17]. Therefore, considering that "staying at home" has been the essential recommendation for individuals with chronic comorbidities, further studies on the necessity of home-based PA during the COVID-19 outbreak are needed.

To the best of our knowledge, no previous reviews on home-based PA in patients with T2DM have been conducted, yet the heterogeneity of home-based PA protocols has been testified [20-22]. On the basis of this evidence, the present study aimed to (1) conduct a systematic search of published studies on home-based PA, (2) map out the characteristics of exercise protocols for home-based PA, (3) evaluate the effects of home-based PA on the investigated outcome measures, and (4) examine the adherence, challenges, and limitations in implementing home-based PA in T2DM patients.

\section{MATERIALS AND METHODS}

\section{Study design}

The protocol of this review has been registered within the Open Science Framework (https://osf.io/3djzp). This scoping review was carried out by using a standardized methodological framework [23]. The identification and screening process for article selection were conducted in accordance with the Preferred Reporting Items for Systematic Reviews and Meta-Analyses extension for Scoping Reviews (PRISMA-ScR) [24].

\section{Search strategy}

Electronic-based searches were performed in six different databases (Scopus, Cochrane Library, SpringerLink, ProQuest, Science Direct, and Google Scholar) to identify studies that describe home-based PA interventions delivered to T2DM patients (Supplement A). A comprehensive search of studies was conducted in January 2021 (updated June 2021). No date restrictions were imposed in the search strategy. The keywords and Boolean operators used in the search were as follows: "home-based physical 
activity" OR "home-based exercise therapy" OR "homebased physical exercise" OR "home-based exercise" OR "home-based exercise training" OR "aerobic exercise" OR "resistance exercise" AND "diabetes mellitus" OR "type 2 diabetes mellitus" AND NOT "type 1 diabetes mellitus" OR "gestational diabetes mellitus".

\section{Eligibility criteria}

Articles or studies that focused on home-based PA and/ or exercise with any design (except systematic and narrative reviews), setting, or duration were included in this study. Studies were excluded if they were (1) not reported in the English language and (2) did not include human subjects.

\section{Study selection}

Two reviewers independently screened all citations and full-text articles retrieved using the above literature search strategy to identify articles that met the eligibility criteria. Discrepancies between reviewers were resolved by consensus. Finally, two reviewers independently extracted data from the selected articles.

\section{Data extraction}

The extracted data from the selected articles are summarized in Table 1. Information regarding the effects of home-based PA on the investigated outcome measures is listed in Table 2.

\section{RESULTS}

A PRISMA-ScR flow diagram showing the stages of article screening and selection is shown in Fig. 1. A total of 443 articles were identified in the search. Briefly, about 342 articles were excluded on the basis of the initial screening of titles and abstracts, leading to the selection of 44 articles that were potentially relevant to the current study. Further screening of the full-text led to the subsequent removal of 34 other articles, resulting in a total of 10 studies that were eligible for data extraction [20-22,2531].

Table 1 shows the characteristics of the included studies. Only seven studies were randomized controlled trials [21,22,25,26,28-30]. In total, 477 T2DM patients were divided into the intervention and control groups, with sample sizes ranging from $n=16$ to $n=40$ and $n=13$ to $\mathrm{n}=44$, respectively (except the study of Shinji et al. [31]). The duration of interventions ranged from 4 weeks to an average of 17.2 months. Four studies included diabetes education, including a healthy eating plan, health counselling, and diabetes self-management prior to the intervention $[21,26,27,31]$. One study further instructed the participants to gradually increase their PA until they achieved 150 minutes per week [27]. Eight studies delivered the exercise demonstration and instruction in the study setting [20-22,25,26,28-30], while two studies used videotape [27] and gave verbal exercise prescription [31] before the intervention.

Aside from supervised sessions at the beginning of the intervention, eight studies included a supervised element either via home visit by the researcher or hospital visit by the participants on more than one occasion throughout the duration of the intervention [20-22,25,26,28-30]. However, two studies were considered unsupervised and maintained contact with their participants by phone or email to keep track of their activities [27,31]. On the other hand, the study of Dunstan et al. [21] implemented two phases of supervised and home-based resistance exercise.

\section{Exercise protocols}

The number of studies that reported on each of the following principles (FITT) is as follows: frequency 10/10, intensity $9 / 10$, time 5/10, and type 10/10. Eight studies followed the standard international guidelines of the FITT principles of exercise protocol, two studies did not follow the recommendation in conducting aerobic exercise, which was limited to 70 minutes per week [20], and did not report the amount of aerobic exercise with frequency of at least 1 day per week [22]. Eight studies prescribed two to five sessions of resistance exercise per week [20-22,25-29]. Five studies prescribed three to six sessions of aerobic exercise per week [26-28,30,31].

Five studies used 1 repetitive maximum (1RM) in identifying the appropriate intensity for resistance exercise and started with $50 \%-60 \%$ of $1 \mathrm{RM}[20,28]$, progressing to $70 \%-95 \%[21,22,29]$. These studies also prescribed two sets of resistance exercise with 8 to 15 repetitions, then progressing to three sets with $90-120$ seconds rest between sets $[20-22,28,29]$. Four studies prescribed moderate-intensity aerobic exercise on basis of the maximum heart rate [20], talk test [26], maximum oxygen uptake 


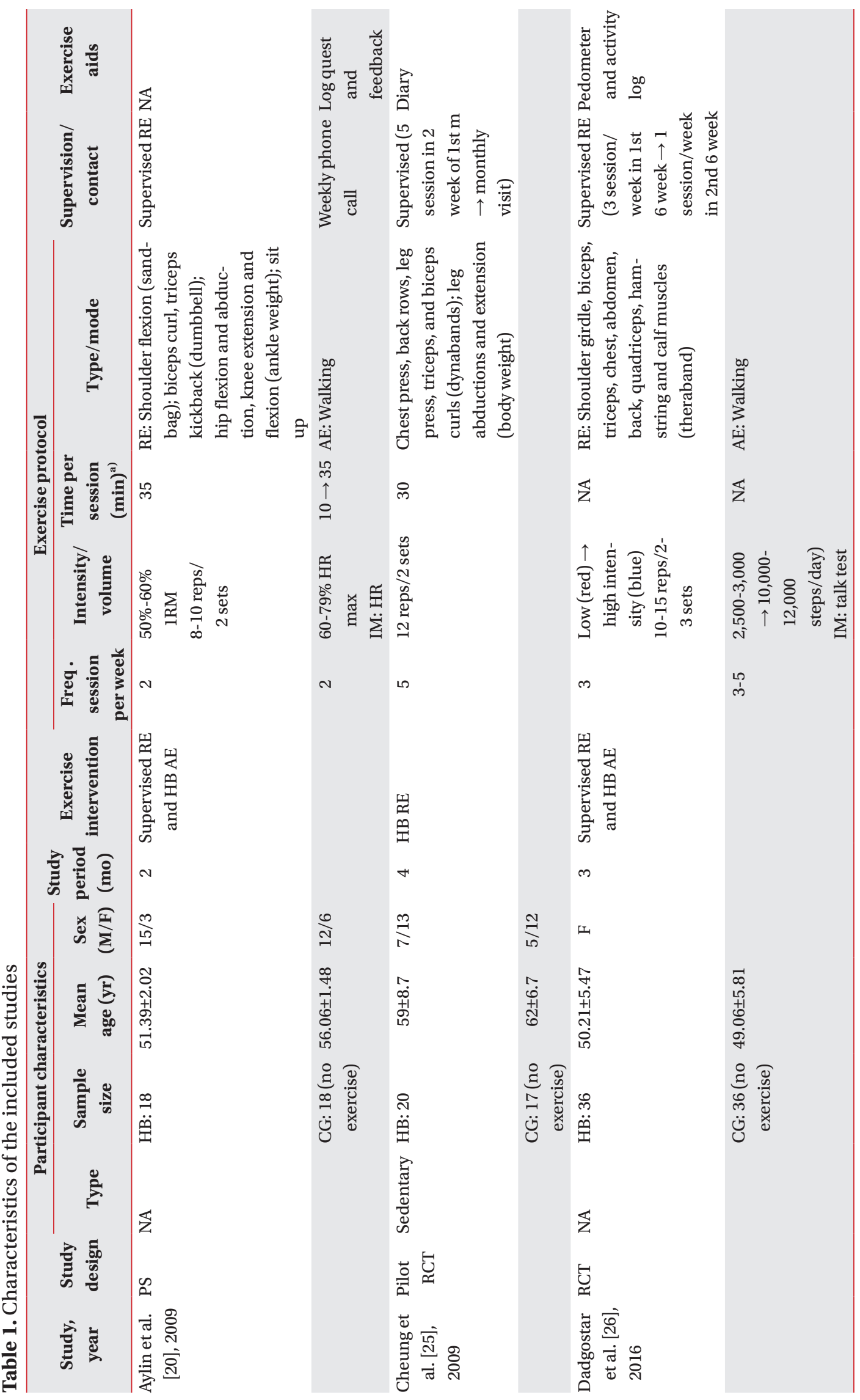




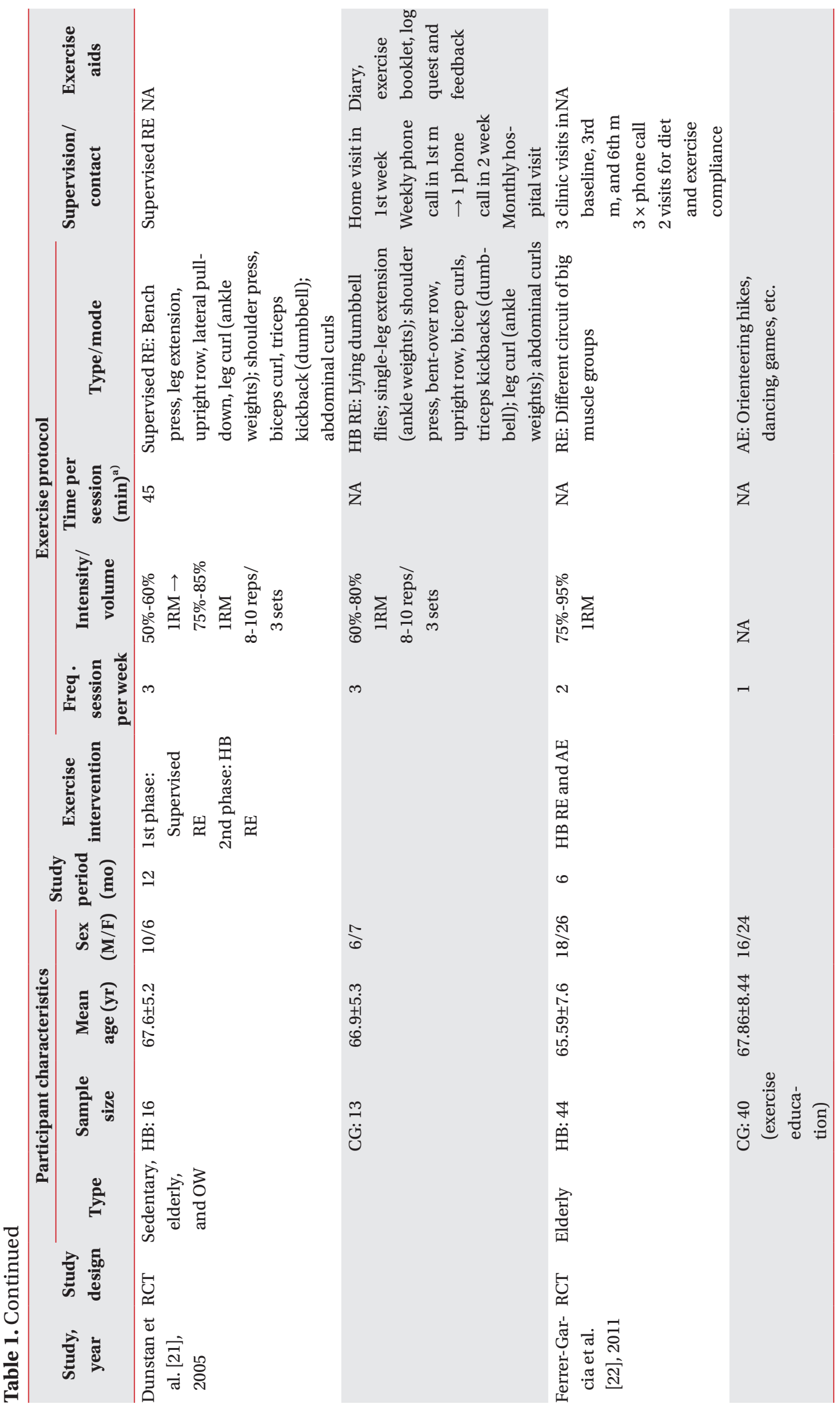




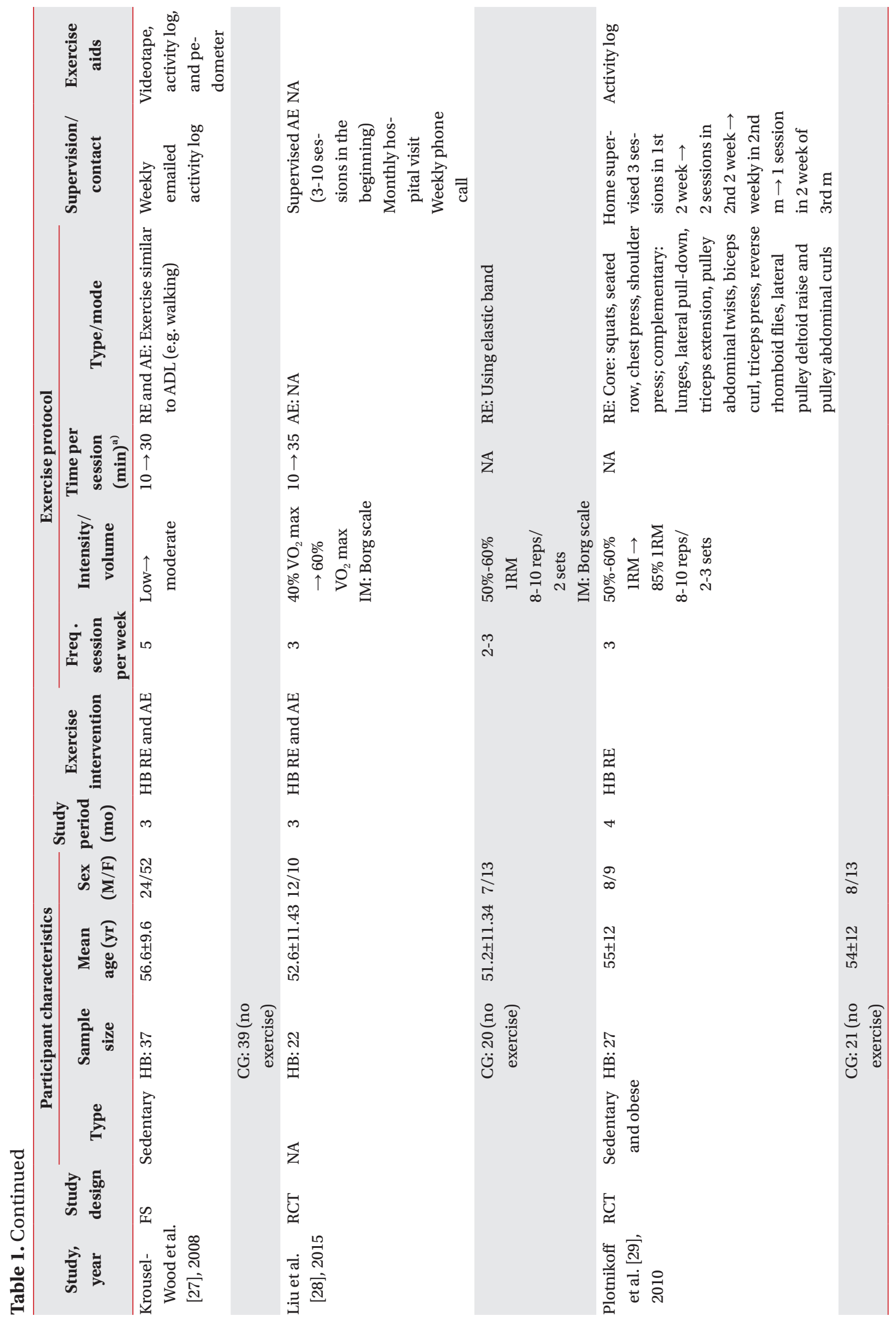



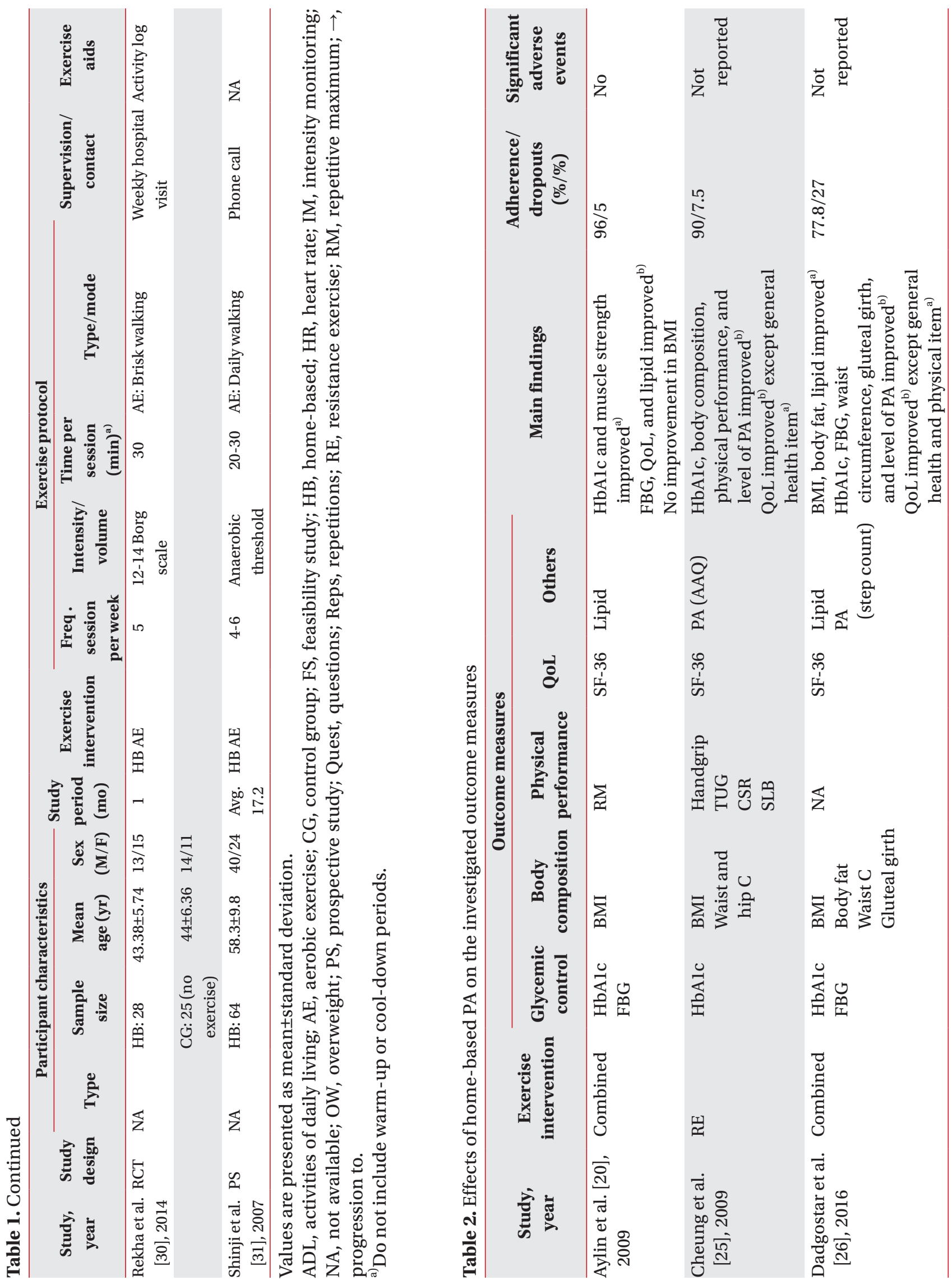


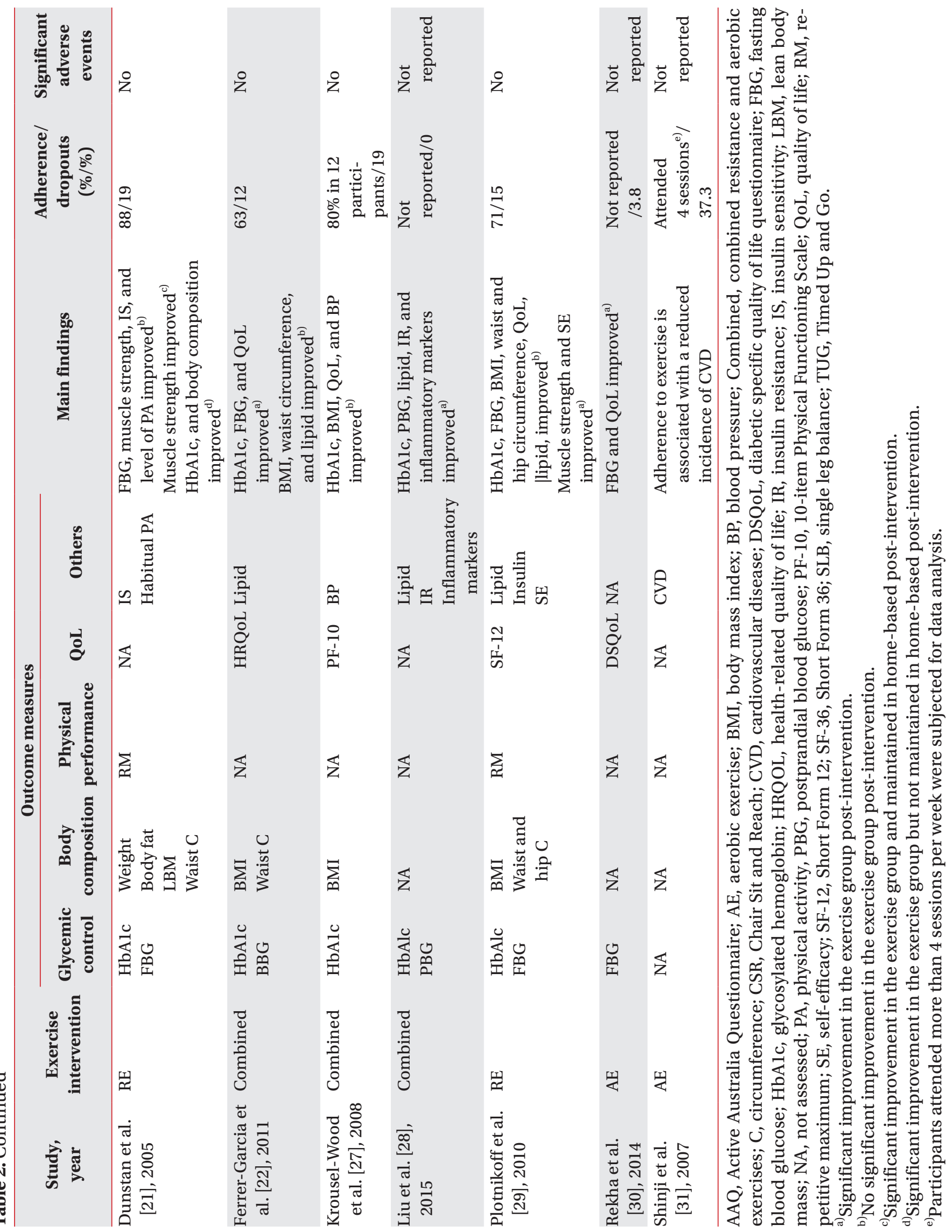




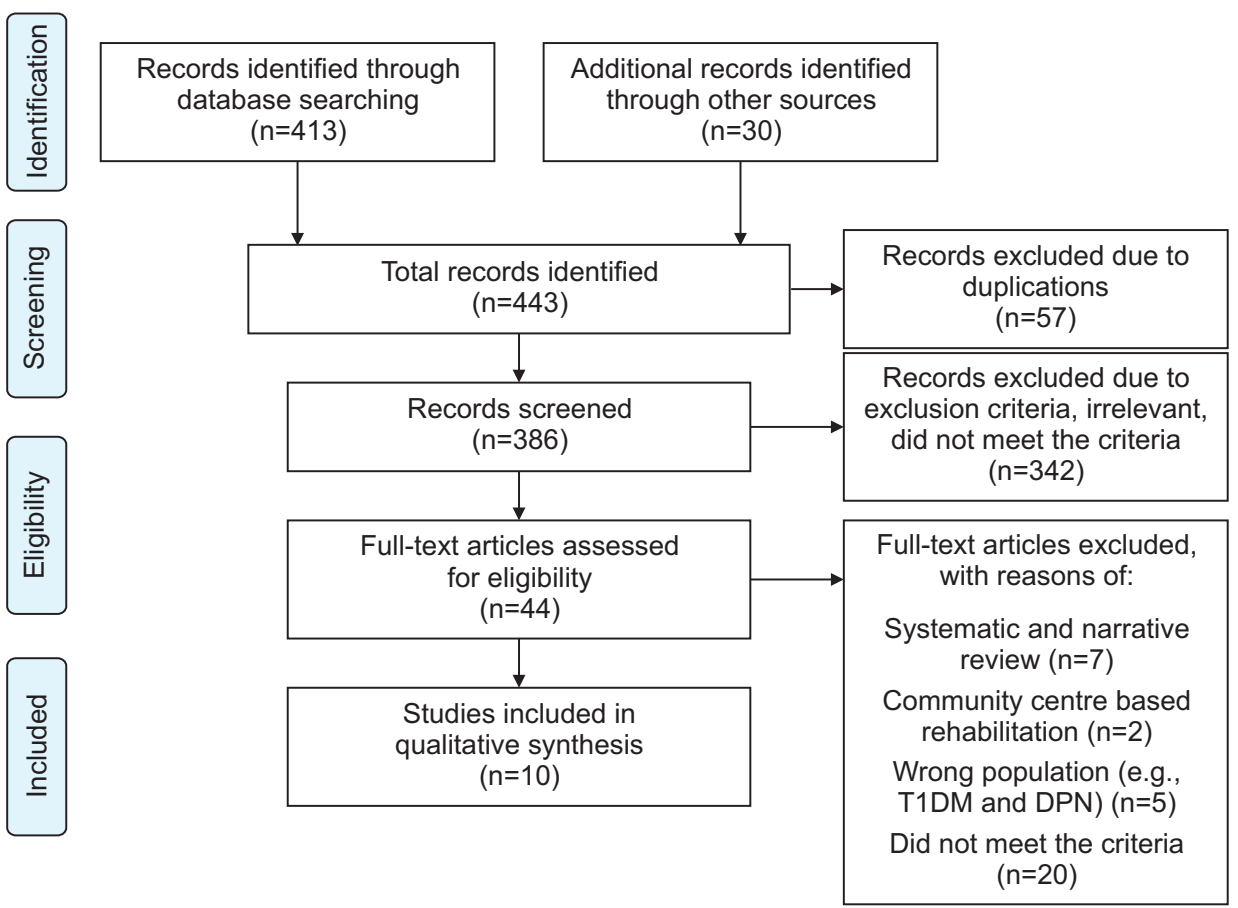

Fig. 1. A Preferred Reporting Items for Systematic Reviews and Meta-Analyses extension for Scoping Reviews (PRISMAScR) flow diagram. T1DM, type 1 diabetes mellitus; DPN, diabetic peripheral neuropathy.
$\left(\mathrm{VO}_{2} \max \right)$, and the Borg scale [28,30]. Meanwhile, one study implemented low- to moderate-intensity aerobic exercise [27]. Shinji et al. [31] adjusted the intensity of aerobic exercise on the basis of the anaerobic threshold. Only one study did not report the intensity of aerobic exercise [22].

In the aspect of time, three studies implemented 30-45 minutes of resistance exercise, excluding warm-up and cool-down $[20,21,25]$. Five studies started with 10-15 minutes of aerobic exercise, excluding warm-up and cool-down, with a gradual increase to 45 minutes at the end of the intervention $[20,27,28,30,31]$. Two studies did not report the time or duration of exercise [22,29].

In the aspect of type, five studies used a combination of resistance and aerobic exercises [20,22,26-28]. Three studies employed resistance exercise only for the intervention $[21,25,29]$. Two studies used aerobic exercise only for the intervention [30,31].

The mode of aerobic exercise varied from walking dependent on the speed $[20,26,27,30,31]$ to orienteering/hiking/dancing [22], which are categorized as unstructured PA. However, one study did not report the mode of aerobic exercise included in the intervention [28]. Six studies incorporated resistance exercise using free weight, such as resistance band, ankle weight, and dumbbell, and own body weight in resistance exercise with standardized frequency and intensity $[20,21,25,26,28,29]$, which are categorized as structured PA. One study did not report the mode of resistance exercise [22].

\section{Effects of home-based PA on the investigated outcome measures}

The most commonly reported outcome measures were the level of glycosylated hemoglobin ( $\mathrm{HbAlc}$ ) and fasting blood glucose (FBG) for glycemic control, body mass index (BMI) and waist and hip circumference for body composition, muscle strength, quality of life (QoL), and level of PA. Four studies showed statistically significant improvement in $\mathrm{HbAlc}$ regardless of combined exercise and resistance exercise alone [20-22,28], and four other studies showed non-significant improvement in HbAlc post-intervention [25-27,29]. However, the study of Dunstan et al. [21] showed a significant improvement in HbAlc during the supervised resistance training, but it was not maintained in home-based resistance training. The study by Rekha et al. [30] is the only one that measured glycemic control using FBG, instead of HbAlc, and found significant improvement in FBG after aerobic exercise.

One study found significant improvement of BMI in combined exercise [26], four studies showed non- 
significant improvement of BMI regardless of combined exercise and exercise alone [22,25,27,29], and one study found no improvement of BMI in combined exercise [20]. The study by Dunstan et al. [21] found significant improvement in body weight during the supervised resistance training and maintained improvement in homebased training. Waist and hip circumference were nonstatistically improved in four studies $[22,25,26,29]$ and two studies $[25,29]$, respectively. The study by Dunstan et al. [21] showed significant improvement of waist circumference during the supervised resistance training, but it was not maintained in home-based training. Muscle strength was examined using 1RM $[20,21,29]$ and handgrip dynamometers [25]. Three studies showed significant improvement in muscle strength after combined exercise and resistance exercise alone $[20,21,29]$. By contrast, one study showed no significant improvement in handgrip strength [25]. The study by Dunstan et al. [21] reported significant improvement in muscle strength during the supervised resistance training and maintained improvement in home-based training. Four studies did not report muscle strength in conducting the resistance exercise [22,26-28].

Several questionnaires were used in assessing the QoL, such as Short Form 12 [29], Short Form 36 (SF36) $[20,25,26]$, Health-Related Quality of Life [22], and Diabetes-Specific Quality of Life questionnaire (DSQoL) [30]. Except for general health and physical items $[25,26]$, five studies demonstrated improvement in QoL although it was not significant [20,25-27-29]. Two studies reported significant improvement in QoL after the combined exercise and aerobic exercise alone $[22,30]$. Metabolic outcomes such as lipid profile and insulin resistance have also been investigated $[20,22,26,28,29]$. Two studies reported significant improvement in lipid profile after combined exercise $[26,28]$, and three studies reported no significant improvement in lipid profile regardless of combined exercise and resistance exercise alone $[20,22,29]$. Three studies assessed the level of PA before the exercise training using the Active Australia Questionnaire [25], self-reported habitual PA [21], and 10-item Physical Functioning Scale [27]. These studies found no significant improvement in the level of PA postintervention $[21,25,27]$. Only one study examined flexibility, balance, and functional mobility using Chair Sit and Reach, Single Leg Balance, and Timed Up and Go, respectively [25]. The study by Shinji et al. [31] evaluated the incidence of cardiovascular disease (CVD) with the adherence of PA. This study found that adherence to exercise is associated with a reduced incidence of CVD.

\section{Adherence, challenges, and limitations}

In general, six studies indicated good adherence of participants with the home-based training ranging from $63 \%$ to $96 \%[20-22,25,26,29]$. One study found that 12 patients $(38 \%)$ in the intervention group achieved at least $80 \%$ or more of the prescribed exercise regardless of the supplemental videotape as an exercise aid [27]. Two studies did not report adherence $[28,30]$. The study by Shinji et al. [31] included participants who attended more than four sessions per week for data analysis.

Additionally, five studies reported no significant adverse events or injuries during the intervention [20$22,27,29]$, five studies did not report the adverse events of home-based PA [25,26,28,30,31]. Nevertheless, Plotnikoff et al. [29] reported that eight patients in the exercise group presented with musculoskeletal injuries of the knee, shoulder, back, and backside that impaired their ability to perform some of that diminished their compliance to the exercise.

Meanwhile, Dadgostar et al. [26] reported challenges and limitations of home-based training, such as loss of motivation because of reduced supervision and consultation throughout the intervention. However, this study further emphasized the usage of exercise aid such as pedometers to improve PA [26]. Additionally, regular telephone calls is an effective strategy in maintaining adherence [22]. Poor education on the specific dietary component was also postulated as one of the challenges and limitations of home-based PA [27]. Three studies did not state the challenges and limitations of home-based training $[28,30,31]$.

\section{DISCUSSION}

This scoping review provided a synthesis of the exercise protocols for home-based PA in patients with T2DM. Overall, the majority of included studies adopted a combination of resistance and aerobic exercises in their intervention. The combination of resistance and aerobic exercises for home-based PA led to improvements in glycemic control, muscle strength, and QoL $[20,22,28]$. 
In most home-based PA studies, resistance exercise was performed in accordance with standardized guidelines (i.e., using free weight and own body weight with a frequency of 2-3 sessions per week at moderate intensity with $8-10$ repetitions for $2-3$ sets) $[20,21,26,28]$. The use of free weight in resistance exercise is highly recommended in home and community settings [32]. We found that enrolment of resistance exercise only in home-based PA is an effective method to increase muscle strength and QoL, but it did not significantly improve glycemic control $[25,26,29]$. This is supported by the fact that resistance exercise induces a hypertrophic response and a shift of muscle-fiber type in exercising muscles [33]. Nevertheless, Cheung et al. [25] found no significant increase in muscle strength, probably because of insufficient measurement for handgrip value and focus of resistance exercise on the back, upper arm, and leg regions. Perhaps 1RM strength assessment using standardized protocols may provide a better consistent measure of strength.

Along with resistance exercise, the majority of studies conducted aerobic exercise at a frequency of three to five times per week at moderate intensity lasting for a minimum of 15 minutes or a maximum of 45 minutes excluding warm-up and cool-down each session $[26-28,30]$. Particularly, walking is the most feasible type of unstructured PA and widely used in the therapeutic regime, especially in obese, middle-aged, and elderly patients with T2DM [12,34]. Performance of aerobic exercise only significantly improved glycemic control. However, adequate frequency and timing of walking are critical in improving the metabolic profile [35]. Likewise, a higher training frequency (at least 3 days per week) or longer durations of exercise can lead to better glycemic control in T2DM patients through greater muscle glycogen usage and glycogen repletion rate [36].

By contrast, we found that the study of Aylin et al. [20] conducted an inadequate amount of aerobic exercise, which resulted in significant glycemic control. This study further postulated that a combination of supervised resistance and home-based aerobic exercise may induce improvement in glycemic control. Similarly, the study by Ferrer-Garcia et al. [22] found that engaging in both resistance and aerobic exercises leads to improved glucose control despite the inadequate frequency of aerobic exercise. Correspondingly, combined aerobic and resistance exercise training may increase the rate of glucose infusion and glucose disposal, which leads to better glycemic control [37]. Thus, our findings support a previous review [38] suggesting that a combination of resistance and aerobic exercise shows more significant benefits of PA in patients with T2DM. We also found a non-significant improvement of lipid profile in the study by Aylin et al. [20]. The low frequency of aerobic exercise may lead to the poor outcome of lipid profile.

In addition, the patient's safety is a vital aspect in the prescription of home-based PA. The present review found that most previous studies did not report any major injuries and adverse events throughout the intervention, which is an encouragement for maintaining homebased PA in T2DM patients [20-22,27]. Despite the good outcomes of home-based PA, most studies reported that patient's adherence and compliance to the interventions are the limitations of home-based training [20$22,25,29]$. This finding is in accordance with the study by Dunstan et al. [21], which found a failure in maintaining the gymnasium-based improvements during the homebased phase. Therefore, the maintenance of supervision and contact and supplementary exercise aid are crucial throughout the intervention to encourage adherence and compliance toward home-based PA.

The lack of demonstration and supervision might also contribute to the poor outcome of home-based training [22]. Therefore, personalized supervision in home-based structured PA is crucial in ensuring that T2DM patients achieve the current international guidelines and exercise protocols [39]. A home visit may also help educate T2DM patients to integrate PA in an unsupervised home environment [40]. In brief, the home-based PA approach in T2DM is highly favorable, and strict actions should be taken to address its limitations and barriers.

Poor education on the prescription of home-based PA by healthcare professionals may also hinder the adherence of home-based PA [41]. Therefore, the recommendation of counselling or detailed education sessions on PA by physicians during follow-up appointments and personalized interventions play an essential part in T2DM management. The prescription of home-based PA should be implemented on the basis of tailored interventions owing to obesity and physical inactivity in most patients with T2DM [16]. Thus, pre-screening for the correct techniques of PA is necessary prior to the implementation of home-based PA.

This study has limitations. First, the statistics comparison of the effects of the exercise methods between com- 
bined exercise and resistance or aerobic exercise alone was limited due to the small number of included papers. Second, we did not conduct a formal evaluation of the methodological quality and distinction of study design of the included studies as the purpose of a scoping review is to provide a more comprehensive overview of the available evidence [42]. However, studies involving diabetes education, health counselling, routine diet guidance, and psychological counselling components may have avoided definitive conclusions about home-based PA alone. Therefore, the majority of studies involving home-based PA utilized multiple therapeutic approaches. It is not easy to design an effective home-based PA particularly in T2DM patients with metabolic disorders. Thus, future studies should adopt transparent and consistent exercise principles in the recommendation of home-based PA related to activities of daily living (ADLs) in T2DM patients with metabolic disorders.

\section{CONCLUSION}

This review summarizes the characteristics of homebased PA in patients with T2DM. This review suggests that exercise protocols for home-based PA should include resistance exercise using free weight and own body weight with a frequency of 2-3 sessions per week at moderate intensity with $8-10$ repetitions for $2-3$ sets, along with aerobic exercise (particularly walking) with a frequency of 3-5 times per week at moderate intensity lasting for a minimum of 15 minutes or a maximum of 45 minutes. The performance of combined resistance and aerobic exercise is highly beneficial for patients with T2DM. More studies regarding home-based PA related to ADLs in T2DM patients with metabolic disorders are warranted.

\section{CONFLICT OF INTEREST}

No potential conflict of interest relevant to this article was reported.

\section{AUTHOR CONTRIBUTION}

Conceptualization: Amirah M. Methodology: Amirah M, Haidzir M. Formal analysis: Amirah M, Haidzir M. Funding acquisition: None. Project administration: Amirah
M, Maria J, Haidzir M. Visualization: Amirah M. Writing - original draft: Amirah M. Writing - review and editing: Amirah M, Maria J, Haidzir M, Alif AL. Approval of final manuscript: all authors.

\section{ACKNOWLEDGMENTS}

The authors thank IIUM and UiTM, Malaysia for funding this research through the IIUM-UMP-UITM Sustainable Research Gollaboration Grant 2020 (SRCG) (IIUM/504/G/14/3/1/1/SRCG20-0049) and Geran Kolaborasi Entiti Penyelidikan UiTM (KEPU) 2021(600-RMC/ KEPU 5/3 (001/2021).

\section{SUPPLEMENTARY MATERIALS}

Supplementary materials can be found via https://doi. org/10.5535/arm.21102.

\section{REFERENCES}

1. Asiimwe D, Mauti GO, Kiconco R. Prevalence and risk factors associated with type 2 diabetes in elderly patients aged 45-80 years at Kanungu District. J Diabetes Res 2020,2020:5152146.

2. Eller-Vainicher C, Cairoli E, Grassi G, Grassi F, Catalano A, Merlotti D, et al. Pathophysiology and management of type 2 diabetes mellitus bone fragility. J Diabetes Res 2020,2020:7608964.

3. World Health Organization W. Classification of diabetes mellitus. Geneva: World Health Organization, 2019.

4. Baynes HW. Classification, pathophysiology, diagnosis and management of diabetes mellitus. J Diabetes Metab 2015,6:1-9.

5. International Diabetes Federation. IDF Diabetes Atlas. 9th ed. Brussels, Belgium: International Diabetes Federation Diabetes Atlas, 2019.

6. Williams R, Karuranga S, Malanda B, Saeedi P, Basit A, Besancon $S$, et al. Global and regional estimates and projections of diabetes-related health expenditure: results from the International Diabetes Federation Diabetes Atlas, 9th edition. Diabetes Res Clin Pract 2020,162:108072.

7. Cooper AR, Sebire S, Montgomery AA, Peters TJ, Sharp DJ, Jackson N, et al. Sedentary time, breaks in 
sedentary time and metabolic variables in people with newly diagnosed type 2 diabetes. Diabetologia 2012,55:589-99.

8. Falconer CL, Cooper AR, Walhin JP, Thompson D, Page AS, Peters TJ, et al. Sedentary time and markers of inflammation in people with newly diagnosed type 2 diabetes. Nutr Metab Cardiovasc Dis 2014,24:956-62.

9. Balducci S, Coccia EM. Sedentariness and physical activity in type 2 diabetes during the COVID-19 pandemic. Diabetes Metab Res Rev 2021,37:e3378.

10. Lim S, Bae JH, Kwon HS, Nauck MA. COVID-19 and diabetes mellitus: from pathophysiology to clinical management. Nat Rev Endocrinol 2021,17:11-30.

11. Duclos M, Oppert JM, Verges B, Coliche V, Gautier JF, Guezennec Y, et al. Physical activity and type 2 diabetes. Recommandations of the SFD (Francophone Diabetes Society) diabetes and physical activity working group. Diabetes Metab 2013,39:205-16.

12. Diabetes Canada Clinical Practice Guidelines Expert Committee, Sigal RJ, Armstrong MJ, Bacon SL, Boule NG, Dasgupta K, et al. Physical activity and diabetes. Can J Diabetes 2018,42 Suppl 1:S54-63.

13. American Diabetes Association. 2. Classification and diagnosis of diabetes: Standards of Medical Care in Diabetes-2020. Diabetes Care 2020,43(Suppl 1):S1431.

14. Thomas N, Alder E, Leese GP. Barriers to physical activity in patients with diabetes. Postgrad Med J 2004,80:287-91.

15. Sanghamitra P, Eunice L, Sandipana P, Shayma D, Pranab M. Type 2 diabetes and physical activity: Barriers and enablers to diabetes control in Eastern India. Prim Health Care Res Dev 2019,20:e44.

16. Duclos M, Dejager S, Postel-Vinay N, di Nicola S, Quere S, Fiquet B. Physical activity in patients with type 2 diabetes and hypertension: insights into motivations and barriers from the MOBILE study. Vasc Health Risk Manag 2015,11:361-71.

17. Grabia M, Markiewicz-Zukowska R, Puscion-Jakubik A, Bielecka J, Nowakowski P, Gromkowska-Kepka K, et al. The nutritional and health effects of the COVID-19 pandemic on patients with diabetes mellitus. Nutrients 2020,12:3013.

18. Rastogi A, Hiteshi P, Bhansali A. Improved glycemic control amongst people with long-standing diabetes during COVID-19 lockdown: a prospective, observa- tional, nested cohort study. Int J Diabetes Dev Ctries 2020:1-6.

19. Ruiz-Roso MB, Knott-Torcal C, Matilla-Escalante DC, Garcimartin A, Sampedro-Nunez MA, Davalos A, et al. COVID-19 lockdown and changes of the dietary pattern and physical activity habits in a cohort of patients with type 2 diabetes mellitus. Nutrients 2020,12:2327.

20. Aylin K, Arzu D, Sabri S, Handan TE, Ridvan A. The effect of combined resistance and home-based walking exercise in type 2 diabetes patients. Int J Diabetes Dev Ctries 2009,29:159-65.

21. Dunstan DW, Daly RM, Owen N, Jolley D, Vulikh E, Shaw J, et al. Home-based resistance training is not sufficient to maintain improved glycemic control following supervised training in older individuals with type 2 diabetes. Diabetes Care 2005,28:3-9.

22. Ferrer-Garcia JC, Sanchez Lopez P, Pablos-Abella C, Albalat-Galera R, Elvira-Macagno L, Sanchez-Juan C, et al. Benefits of a home-based physical exercise program in elderly subjects with type 2 diabetes mellitus. Endocrinol Nutr 2011,58:387-94.

23. Arksey H, O'Malley L. Scoping studies: towards a methodological framework. Int J Soc Res Methodol 2005,8:19-32.

24. Tricco AC, Lillie E, Zarin W, O'Brien KK, Colquhoun H, Levac D, et al. PRISMA Extension for Scoping Reviews (PRISMA-ScR): checklist and explanation. Ann Intern Med 2018,169:467-73.

25. Cheung NW, Cinnadaio N, Russo M, Marek S. A pilot randomised controlled trial of resistance exercise bands in the management of sedentary subjects with type 2 diabetes. Diabetes Res Clin Pract 2009,83:e6871.

26. Dadgostar H, Firouzinezhad S, Ansari M, Younespour S, Mahmoudpour A, Khamseh ME. Supervised groupexercise therapy versus home-based exercise therapy: their effects on Quality of Life and cardiovascular risk factors in women with type 2 diabetes. Diabetes Metab Syndr 2016,10(2 Suppl 1):S30-6.

27. Krousel-Wood MA, Berger L, Jiang X, Blonde L, Myers L, Webber L. Does home-based exercise improve body mass index in patients with type 2 diabetes? Results of a feasibility trial. Diabetes Res Clin Pract 2008,79:230-6.

28. Liu Y, Liu SX, Cai Y, Xie KL, Zhang WL, Zheng F. Effects of combined aerobic and resistance training on 
the glycolipid metabolism and inflammation levels in type 2 diabetes mellitus. J Phys Ther Sci 2015,27:236571.

29. Plotnikoff RC, Eves N, Jung M, Sigal RJ, Padwal R, Karunamuni N. Multicomponent, home-based resistance training for obese adults with type 2 diabetes: a randomized controlled trial. Int J Obes (Lond) 2010,34:1733-41.

30. Rekha K, Anandh V, Rajendran K. Home based therapeutic intervention for type 2 diabetes mellitus. J Chem Pharm Sci 2014,7:275-80.

31. Shinji S, Shigeru M, Ryusei U, Mitsuru M, Shigehiro $\mathrm{K}$. Adherence to a home-based exercise program and incidence of cardiovascular disease in type 2 diabetes patients. Int J Sports Med 2007,28:877-9.

32. McGinley SK, Armstrong MJ, Boule NG, Sigal RJ. Effects of exercise training using resistance bands on glycaemic control and strength in type 2 diabetes mellitus: a meta-analysis of randomised controlled trials. Acta Diabetol 2015,52:221-30.

33. Marwick TH, Hordern MD, Miller T, Chyun DA, Bertoni AG, Blumenthal RS, et al. Exercise training for type 2 diabetes mellitus: impact on cardiovascular risk: a scientific statement from the American Heart Association. Circulation 2009,119:3244-62.

34. Klimek M, Knap J, Masternak M, Reda M. Physical activity in prevention and treatment of type 2 diabetes mellitus. J Educ Health Sport 2019,9:1175-81.

35. Moghetti P, Balducci S, Guidetti L, Mazzuca P, Rossi E, Schena F, et al. Walking for subjects with type 2 diabe- tes: a systematic review and joint AMD/SID/SISMES evidence-based practical guideline. Nutr Metab Cardiovasc Dis 2020,30:1882-98.

36. Oliveira C, Simoes M, Carvalho J, Ribeiro J. Combined exercise for people with type 2 diabetes mellitus: a systematic review. Diabetes Res Clin Pract 2012,98:187-98.

37. Cuff DJ, Meneilly GS, Martin A, Ignaszewski A, Tildesley HD, Frohlich JJ. Effective exercise modality to reduce insulin resistance in women with type 2 diabetes. Diabetes Care 2003,26:2977-82.

38. Ginszt A, Ginszt M, Majcher P, Tarkowski Z. Effects of exercise on blood glucose levels in type 2 diabetic patients: literature review. Polish Ann Med 2018,25:2726.

39. Esteves M, Gouveia A, Rodrigues R, Pinheiro P, Bras R, O'Hara K, et al. Supervised exercise patterns among diabetic and non-diabetic Portuguese adults. Ann Appl Sport Sci 2019,7:49-56.

40. Karatay G, Akkus Y, Ozturk B, Sulu B. The effectiveness of a home-based diabetes management program on diabetes control. Arch Prev Med 2016,1:015-020.

41. Advika TS, Idiculla J, Kumari SJ. Exercise in patients with type 2 diabetes: facilitators and barriers: a qualitative study. J Family Med Prim Care 2017,6:288-92.

42. Peters MD, Godfrey CM, Khalil H, McInerney P, Parker D, Soares CB. Guidance for conducting systematic scoping reviews. Int J Evid Based Healthc 2015,13:1416. 